\title{
Un homme politique désœuvré: Machiavel, historien et penseur politique
}

GÉRALD

ALLARD

Summary: A philosopher who deeply influenced Western history, Machiavelli was, as well, involved in the affairs of his time. This paper aims to analyze the pragmatic context of Machiavelli's production, for instance, his political exile from Florence. In order to draw the lines of this "praxis," contemporary biographies, letters of Machiavelli and, of course, the books (The Prince, Discourses, History of Florence) are revisited and reread in each other's light. This approach allows one to appreciate more the "revolutionary" and "anticlerical" dimensions of Machiavelli's philosophy.

$\mathrm{D}$ 1498 à 1512, Nicolas Machiavel est secrétaire de la Seconde Chancellerie de la République de Florence, État fondé sur une opposition farouche aux Médicis. En 1513, les Médicis, revenus au pouvoir, font arrêter, emprisonner et torturer l'ancien secrétaire qu'ils croient mêlé à une conspiration contre leur nouveau régime. Relâché après quelques mois, Machiavel est empêché, pendant de longues années, de retourner dans le jeu politique de sa ville, et ce par ordre des Médicis. Il ne peut rien faire, mais il peut parler : comme il le dit dans une lettre à François Vettori, « je ne sais discourir ni sur l'art de la soie, ni sur l'art de la laine, ni sur les gains et les pertes d'argent ; il m'appartient donc de discourir sur l'État, puisque je dois ou bien faire vœu de me taire ou bien de parler de ce seul sujet ${ }^{1}$. Et dans les faits il parle abondamment de l'État dans des lettres adressées à Vettori et à d'autres ; mais il en parle aussi lors de rencontres qui ont lieu aux Jardins Oricellari avec des jeunes hommes comme Zanobe Buondelmonti et Côme Rucellai. Plus important, Machiavel ne se satisfait pas de parler de politique : ces conversations se transforment en écrits comme Le Prince et les Discours sur la première décade de Tite-Live, évidemment, mais aussi l'Art de la guerre et l'Histoire de Florence. Car Machiavel, comme tout le monde le 
sait, est un des penseurs-écrivains les plus intéressants de la Renaissance, un maître qui a laissé derrière lui quelques chefs-d'œuvre, comme autant de galets pour que les Petits Poucets que nous sommes puissent se retrouver sur les sentiers de la vie. Qu'en est-il de cet homme désœuvré en mal d'œuvres politiques ? Qu'en est-il en même temps du désœuvrement à l'origine de ses œuvres ? Les détails historiques suivants introduiront dans l'atelier machiavélien et prépareront à une réflexion plus poussée sur l'historien et penseur Machiavel.

Dans une lettre adressée à Machiavel, Zanobe Buondelmonti, un des jeunes interlocuteurs de Machiavel aux Jardins Oricellari, offre la première critique d'une œuvre qui sera publiée avec le Prince, La Vie de Castruccio Castracani, sorte de reprise sur le mode biographique du livre le plus connu de Machiavel. Buondelmonti écrit : " Nous l'avons lue, Luigi, Guidetto, Diacettino, Antonfrancesco et moi, et nous l'avons examinée un peu ensemble. En général, nous avons conclu qu'elle était bonne et bien faite. Mais on note quelques passages qui, tout en se défendant bien, pourraient néanmoins être améliorés : par exemple, la dernière partie faite des bons mots, des traits d'esprit et des saillies de Castruccio. Cette partie pourrait être meilleure si elle était raccourcie, parce qu'en plus du fait qu'il y a trop de ces mots et saillies, une partie d'entre eux ont été attribués à d'autres sages anciens et modernes $\gg^{2}$. Le jeune homme a tout à fait raison : sur les trente-quatre traits que Machiavel met dans la bouche de Castruccio, trente et un viennent du traité les Vies des philosophes illustres de Diogène Laërce.

Or l'auteur de La Vie de Castruccio Castracani n'a pas tenu compte de ce que lui avaient dit ses disciples, puisque le texte final semble avoir conservé intacte la partie qui les avait heurtés. Il est donc permis de croire que les jeunes interlocuteurs du vieux maître n'étaient pas à ses yeux des esprits critiques sans faille, puisqu'il n'a pas tenu compte de leur avis. Pourtant dans la lettre dédicatoire des Discours sur la première décade de Tite-Live Machiavel dit de lui-même qu'il n'a que peu de talent et que Zanobe est de ces « hommes qui par leurs qualités mériteraient d'être des princes $»^{3}$. Tout le monde sait que Machiavel avait peu d'estime pour le destinataire officiel du Prince ; quand il s'agit de Laurent de Médicis, tout le monde sait que Machiavel était capable d'une ironie meurtrière. Mais alors qu'en est-il au juste des destinataires des Discours?

De plus, si Machiavel est l'apologiste de la verità effettuale della cosa, la vérité effective de la chose, vérité effective née du regard impitoyable posé sur les faits et produisant d'impitoyables leçons efficaces, il est aussi un lecteur, un maître lecteur, un homme qui jette les yeux dans des livres et qui tire de là aussi des leçons magistrales. Il les tire donc ces leçons, mais en les tirant par les cheveux, comme on dit. Car, pour prendre l'exemple de 
la Vie de Castruccio Castracani, non seulement les traits d'esprit des philosophes anciens sont attribués à tort à Castruccio, mais aussi ils sont transformés pour les rendre plus machiavéliens.

Un passage de cette biographie servira d'illustration. Comme on avait demandé à Castruccio si pour sauver son âme il avait jamais pensé se faire frère, il répondit que non, parce qu'il lui paraissait étrange que frère Lazzero — un prêtre naïf - doive aller en paradis et Ugoccione della Faggiola un grand militaire - en enfer. La remarque est une adaptation d'un mot d'esprit de Diogène le Cynique : Machiavel fait passer le lecteur du monde peuplé par les dieux païens au monde de la foi chrétienne. Il semblerait que les lectures pré-chrétiennes de Machiavel aient fait de l'auteur, ou du moins de son personnage, un homme bien peu respectueux de la religion de son temps. Quoi qu'il en soit, les livres ont un grand rôle à jouer dans l'œuvre de pensée de l'homme politique désœuvré.

Servira de preuve un autre passage tiré de la correspondance de Machiavel, soit d'une lettre écrite par son ami et confrère de bureau, Biagio Buonaccorsi. À la date de l'envoi de la lettre, Machiavel est en ambassade à Imola pour la république de Florence auprès de César Borgia. Dans quelques jours, ce dernier assassinera de façon spectaculaire quelques-uns de ses adversaires politiques; Machiavel verra tout et racontera tout dans le célèbre chapitre septième du Prince et dans le court opuscule au titre interminable, Description de la façon dont le duc Valentinois s'y est pris pour tuer Vitellozzo Vitelli, Oliveretto da Fermo, le seigneur Paul et le duc de Gravina Orsini. Montesquieu le premier a enseigné aux lecteurs du Prince que César Borgia est l'exemple par excellence de Machiavel ; il est son $«$ héros $»^{4}$. Avec cette lettre de Biagio Buonaccorsi, nous sommes donc placés, pour ainsi dire, dans le laboratoire du Prince. Or dans la lettre de Biagio à son copain de bureau Nicolas, on parle de tout et de rien, mais au milieu de ce rien, il y a ces mots : "Nous avons fait chercher les Vies de Plutarque, et l'on n'en trouve pas à vendre à Florence. Prends patience, parce qu'il faut écrire à Venise. Et à vrai dire, tu peux aller te faire pendre avec tes nombreuses demandes $»^{5}$. Il paraît donc que Machiavel, qui est en mission auprès de César Borgia depuis quelques mois, a demandé à son ami de lui envoyer des livres, rien de moins que les Vies des hommes illustres de Plutarque ; et il y a fort à parier qu'à l'époque où il examinait les crimes impies du fils du pape Alexandre VI, il lisait les faits et gestes des héros de Plutarque. Or dans le Prince, Machiavel corrige, discrètement, l'avis de Plutarque sur Philopœmen, un des hommes illustres dont il raconte la vie : plutôt que de croire, comme le voulait le sage Grec, que Philopœmen paraissait s'intéresser plus que de raison à la gloire militaire ${ }^{6}$, l'habile Florentin affirme, à partir du même exemple, qu' « un prince doit n' avoir nul 
autre objet ni autre pensée, ni prendre pour discipline que l'art de la guerre, ses institutions et son école $\gg^{7}$. Il est tentant de croire que cette conclusion fut tirée en regardant agir César Borgia sans doute, mais aussi en lisant les Vies des hommes illustres de Plutarque et que l'observateur de la vérité effective était aussi un lecteur attentif de « ceux qui ont écrit sur la conduite d'un prince envers ses sujets et ses amis $»^{8}$. Machiavel savait faire dialoguer le fait avec la littérature, éclairant l'un par l'autre et l'autre par l'un.

En revanche, il y a une autre forme de dialogue que Machiavel affectionnait. Au chapitre troisième du Prince, Machiavel discute des erreurs que peut faire un prince nouveau, qui cherche à imprimer sur un peuple le sceau de son autorité. Il traite là, entre autres, des tactiques qu'avait utilisées le roi de France lorsqu'il avait tenté de conquérir l'Italie du temps de César Borgia. Machiavel écrit : " Je parlai de cette matière à Nantes avec le cardinal de Rouen. Comme le cardinal de Rouen me disait que les Italiens ne comprenaient pas [la conduite] de la guerre, moi je lui répondis que les Français ne comprenaient pas [la conduite] de l'État, parce que s'ils la comprenaient, ils ne laisseraient pas l'Église en arriver à tant de grandeur ». On imagine la scène, le petit secrétaire florentin affrontant le grand cardinal français sur la question de la conquête de l'Italie et surtout sur celle de la meilleure façon de gérer les choses politiques : on entend là un dialogue de fond sur le problème théologico-politique, lequel définit presque la Renaissance. Or tout de suite après, Machiavel, l'homme d'État, ajoute ce sur quoi il s'appuyait pour rétorquer ainsi à l'homme d'Église : c'est rien de moins que la célèbre vérité effective. «Et l'on a vu par expérience qu'en Italie la grandeur de l'Église et de l'Espagne a été causée par la France, dont s'ensuivit sa propre perte. D'où l'on tire une règle générale, qui ne souffre jamais ou rarement d'exceptions, d'après laquelle celui qui est cause que quelqu'un devienne puissant se perd. »Cette apologie passionnée de la puissance rationnelle humaine qui dirige l'État, puissance rationnelle qui prétend savoir envers et contre les prétentions de l'Église et donc de la foi, cette apologie est un des thèmes essentiels de Machiavel. Il ajouterait qu'elle est essentielle de toute pensée vraiment libre.

Ces anecdotes présentent Machiavel en tant qu'homme d'action, homme d'écriture et homme de réflexion. Or cet homme se révèle grand selon chacun de ces vecteurs. Sans doute Niccolò Machiavelli, fils de Bernardo Machiavelli, né en 1469, mort en 1527, est-il, comme nous tous, un agent de l'histoire : nous sommes fils et filles de notre époque, pris dans ses limites, poussés et tirés par ses exigences, accomplis par les possibilités qu'elle nous offre et que nous réalisons, frustrés par celles qui nous échappent. Pourtant, sur ce plan, Machiavel est un être privilégié. Au moins pour la raison qu'il vivait durant une période particulièrement intéressante, la 
Renaissance, et dans une cité qui est comme la quintessence de la Renaissance, Florence, Firenze città delle arti, comme disent encore aujourd'hui les fiers Florentins 9.

Par ailleurs, cette période et cette ville, Machiavel pouvait et devait les connaître d'une façon toute particulière en raison du poste de secrétaire de la seconde chancellerie de la république qu'il occupa de 1498 à 1512 . Et ce d'autant plus qu'à la longue l'irrésistible secrétaire fit de son poste de subalterne payé par l'État une position de conseiller politique influent, voire le plus influent, du gonfalonier, ou chef d'État, Soderini ${ }^{10}$.

Ce n'est donc pas un intellectuel que le pape Clément VII, né Jules de Médicis, avait chargé d'écrire l'histoire de Florence : Nicolas Machiavel était un homme d'expérience et de métier qui traitait de ce qu'il connaissait bien, soit l'histoire de sa cité. Or comme le montre la lettre dédicatoire de l'Histoire de Florence, l'homme politique désœuvré s'était fait historien en épousant les obligations de la discipline. "Car dans tous mes récits, écrit-il, je n'ai jamais voulu recouvrir une action malhonnête au moyen d'une honnête cause, ni noircir une œuvre louable comme si elle avait été faite pour une fin contraire $»^{11}$. Après lecture de l'œuvre, on a toutes les raisons de reconnaître que Machiavel est, avant Guicciardini son ami, un grand historien : il est le Thucydide, ou peut-être l'Hérodote, de la Renaissance. C'est là un deuxième lien qu'il entretient avec l'histoire.

La relation à la discipline de l'histoire, Machiavel l'avait déjà éprouvée dans ce que plusieurs considèrent son chef-d'œuvre : les Discours sur la première décade de Tite-Live. Car ce traité de politique se présente au lecteur comme des réflexions sur la matière qu' offrait l'historien latin Tite-Live, ou encore comme un commentaire de l'histoire de Rome. Mais il y a commentaire et commentaire. Dès le proème du premier livre, Machiavel fait comprendre qu'il a réfléchi sur la discipline de l'histoire, sur son but, et qu'il y a réfléchi à partir de ce qu'il est ou a été, un homme d'action. Car, pour lui, l'histoire n'est pas une discipline libérale comme d'autres, mettons la philosophie ou la littérature, et les discorsi, ou essais, sur Tite-Live qu'il entreprend ne sont pas l'occupation d'un oisif, ni même d'un exilé de la vie politique ; pour l'ancien secrétaire de la république, l'essentiel, soit la vie politique de son temps, était en jeu lorsqu'il lisait ce qui se fit autrefois dans la république de Rome :

Voyant que les actions les plus vertueuses que nous montrent les histoires, actions qui furent accomplies par des royaumes et des républiques antiques, par des rois, des capitaines, des citoyens, des législateurs et d'autres qui se sont fatigués pour leur patrie, sont plutôt admirées qu'imitées et sont même si totalement évitées de tous qu'il ne nous reste aucune trace de cette antique vertu, je ne puis pas ne pas en éprouver de l'étonnement ainsi que de la douleur. ${ }^{12}$ [. . .] Je crois que cela [soit la stérilité de l'étude 
historique] ne naît pas tant de l'état de faiblesse où la religion actuelle a conduit le monde ou du mal qu'un loisir ambitieux a fait dans un grand nombre de provinces et de cités chrétiennes, que du fait qu'on n'a pas une véritable connaissance de l'histoire pour en tirer, par la lecture, le sens qui s'y trouve et en goûter la saveur. ${ }^{13}$

En somme, Machiavel fait œuvre d'historien ou réfléchit sur l'histoire, ou commente l'œuvre d'un historien, parce que le passé, quand il est bien examiné, éclaire le présent et l'avenir, parce que l'histoire lue peut servir à l'histoire à faire. L'étude de l'histoire est, pour Machiavel, un acte politique $^{14}$.

Cela n'est pas moins vrai du livre qui a rendu Machiavel célèbre pour tous les temps. Car comme les Discours, le Prince est une réflexion sur la matière historique. Aussi il y a quelque chose de touchant, et peut-être de comique, àvoir Machiavel s'approcher de la famille des Médicis dans la personne de Laurent de Médicis avec comme tout présent, non pas un cheval ou une pierre précieuse, comme font les autres, mais un livre tout maigre, un opuscule de vingt-six chapitres ${ }^{15}$ : «Désirant donc m'offrir à Votre Magnificence avec quelque témoin de ma servitude envers Elle, je n'ai pas trouvé, parmi mes affaires, quelque chose qui me soit plus cher ou que j'estime autant que la connaissance des actions des grands hommes, apprise par une longue expérience des choses modernes et une lecture continuelle des anciennes : ayant réfléchi sur elles et les ayant examinées longuement et avec diligence, puis les ayant réduites en un petit volume, je les envoie à Votre Magnificence » ${ }^{16}$. Le Prince est donc le fruit de l'histoire, c'est-à-dire à la fois des faits dont se souvient un homme d'action devenu un homme désœuvré, et des faits tirés de l'étude de l'histoire; tel que Machiavel le présente, il n'est rien de plus que le distillé de son expérience historique directe et indirecte. Car, répétons-le, pour lui, le passé, et même le passé lointain, aide à comprendre le présent et éclaire l'avenir.

Qui douterait encore trouvera dans le texte même du Prince, une preuve suffisante de l'importance de l'histoire et de l'étude de l'histoire pour l'auteur. Parlant du prince en tant que chef militaire, Machiavel écrit :

\footnotetext{
Mais quant à l'exercice de l'esprit, le prince doit lire les histoires et considérer en elles les actions des hommes excellents : voir comment ils se sont gouvernés dans les guerres ; examiner les causes de leurs victoires et de leurs pertes, pour pouvoir éviter celles-ci et imiter celles-là ; surtout, faire comme ont fait par le passé plusieurs hommes excellents qui ont choisi d'imiter une personnalité qu'on a louée et glorifiée, gardant toujours devant eux ses gestes et ses actions, comme on dit qu'Alexandre le Grand imitait Achille ; César, Alexandre ; Scipion, Cyrus. ${ }^{17}$
}

Pour Machiavel, les leçons de l'histoire ne sont pas d'abord techniques : il ne s'agit pas seulement d'apprendre des erreurs des autres et d'imiter les 
solutions qu'ils ont inventées aux problèmes qu'ils ont affrontés et qu'on risque d'affronter à son tour. Car l'histoire éduque bien plus qu'elle n'instruit : les grands hommes du passé ont appris à être grands en examinant la vie des grands hommes qui les ont précédés; et une des leçons de l'histoire est que l'histoire forme le cœur des hommes ${ }^{18}$. Aussi les écrivains qui racontent la vie des Achille et l'histoire des Alexandre et des Cyrus sont les éducateurs de l'humanité. Mais alors Machiavel s'imaginerait-il le Homère, le Plutarque et le Xénophon de l'Italie et ainsi l'éducateur de Laurent et des autres princes ? L'hypothèse n'a rien d'impossible. D'ailleurs, l'auteur en avoue à demi la justesse dans la phrase qui suit : "Quiconque lira la vie de Cyrus, écrite par Xénophon, reconnaîtra ensuite, dans la lecture de celle de Scipion, combien cette imitation servit à sa gloire et combien Scipion se conforma pour la chasteté, l'affabilité, l'humanité, la générosité à ce que Xénophon a écrit au sujet de Cyrus ». Ce que Xénophon fit en écrivant l'Éducation de Cyrus - c'est le sens du titre traditionnel Cyropédie Machiavel se voit le faire en écrivant le Prince ${ }^{19}$.

Machiavel est-il, à ses propres yeux, un homme d'action, qui s'est adonné à la réflexion quand la fortune l'a réduit au désœuvrement? Ou un homme de pensée qui est digne de mémoire parce qu'il a osé dire quelque chose que personne d'autre n'avait dit avant lui ${ }^{20}$ ?

Comme l'ont signalé bien des commentateurs, le traité Des principautés, comme il devait se nommer ${ }^{21}$, est de structure bizarre, au moins parce qu'il est construit sur deux thèmes qui se suivent sans nécessairement se fondre pour former un tout. Les quatorze premiers chapitres sont une sorte de manuel politique où l'on analyse les espèces de principautés et l'art militaire nécessaire pour les acquérir et les conserver. Au quinzième chapitre, Machiavel recommence son livre en dressant la liste des qualités d'un prince, qualités qu'il exposera et développera jusqu'au chapitre vingt-troisième. Or ce quinzième chapitre commence par ce qu'on a appelé un cri :

Comme moi je sais que beaucoup d'hommes ont écrit là-dessus, je crains d'être tenu pour présomptueux en écrivant moi aussi là-dessus, en me démarquant des institutions des autres surtout pour discuter de cette matière-ci. Mais comme mon intention est d'écrire quelque chose d'utile pour qui le comprendra, il m'a paru plus convenable de suivre la vérité effective de la chose que l'imagination qu'on a d'elle. Beaucoup d'hommes se sont imaginé des républiques et des principautés qu'on n'a jamais vues ni jamais connues existant dans la réalité ; mais une telle distance sépare la façon dont on vit de celle dont on devrait vivre que celui qui met de côté ce qu'on fait pour ce qu'on devrait faire apprend plutôt à se perdre qu'à se préserver ; parce qu'un homme qui veut faire profession d'être tout à fait bon, il faut qu'il se perde parmi tant d'hommes qui ne le sont pas. C'est pourquoi il est nécessaire à un prince qui veut se maintenir d'apprendre à pouvoir n'être pas bon et de se servir ou non de ce savoir selon la nécessité. Laissant 
donc de côté les choses imaginées au sujet d'un prince et examinant celles qui sont vraies, je dis $\left[\ldots . .^{22}\right.$

Notons d'abord la construction binaire des phrases. Machiavel met d'un côté l'imagination, le devoir être et l'échec, de l'autre la vérité, le fait et l'efficacité. Le lecteur est placé devant un choix à faire : conduit par son guide, il se trouve soudain à la fourche du chemin de la vie. On ne saurait poser plus dramatiquement le problème existentiel. De plus, et comme pour donner un lieu politique à la première triade, Machiavel parle de républiques imaginaires et de principautés invisibles. Les régimes politiques proposées par les prédécesseurs de Machiavel sont, sans doute, la république en paroles que le Socrate de Platon invente lors d'une conversation avec des jeunes hommes et, en deuxième lieu, le royaume des cieux promis par le Christ à ceux qui le suivraient ${ }^{23}$. Enfin, et de façon plus importante, Machiavel se présente comme l'adversaire de ceux qui l'ont précédé et qui de son avis se sont trompés ${ }^{24}$. Or le face à face qu'il propose est un face à face entre écrivains ou entre livres : Machiavel répond par écrit à ce que les autres ont écrit avant lui ; il s'adresse à un lecteur qui a à apprendre de lui le contraire de ce qu'il apprendrait ailleurs ; Machiavel enseigne pour qu'on apprenne, mais aussi pour qu'on comprenne. En somme, Machiavel écrit pour avertir et convertir, mais pour ce faire il doit pervertir, c'est-à-dire renverser les royaumes et principautés imaginaires qui n'existent que dans les cerveaux des autres. En écrivant le Prince, Machiavel se voit comme un prince conquérant, un prince qui conquiert les âmes ${ }^{25}$.

L'expression paraîtra exagérée ${ }^{26}$. Mais voici un autre passage de lui, qui parle de terre à conquérir : l'auteur du Prince, l'auteur des Discours sur la première décade de Tite-Live est le découvreur d'un nouveau monde moral ; il est non pas un Moïse qui conduit son peuple aux frontières de la Terre promise, mais un Christophe Colomb qui découvre et révèle à ses concitoyens un monde qui n'existait pas pour eux parce qu'ils ne le connaissaient pas. Le livre commence à peu près comme suit :

La nature envieuse des hommes a toujours rendu aussi dangereux de trouver des façons et des institutions nouvelles que de chercher des mers et des terres inconnues. Car ils sont plus prompts à blâmer les actions des autres qu'à les louer. Néanmoins, poussé par le désir naturel, qui a toujours été en moi, de faire sans aucune crainte ces choses qui, je crois, apportent un bénéfice commun à chacun, j'ai décidé d'entrer sur une voie, laquelle, comme personne n'y a jamais cheminé, même si elle m'apportera ennui et difficulté, pourrait aussi me procurer une récompense de la part de ceux qui considéreraient humainement la fin de mes efforts. ${ }^{27}$

Dans le contexte des Discours, la découverte de ce chemin nouveau, ou de ces terres intellectuelles, se fera par le commentaire de Tite-Live sans doute, 
et donc par un retour en arrière. C'est là souligner que le Grand Secrétaire se présente comme un docteur ou un professeur ${ }^{28}$. Et ce docteur craint d'être envié parce qu'il propose ce que personne n'a jamais connu avant lui, ce que personne n'a jamais enseigné avant lui. Encore une fois, Machiavel se voit comme un éducateur, comme il le dit explicitement au proème de la deuxième partie : "C'est le devoir d'un homme bon d'enseigner aux autres ce bien que par la malignité des temps et de la fortune tu n'as pas pu faire, afin que, comme un grand nombre en deviendra capable, quelqu'un d'entre eux, plus aimé du ciel, puisse le faire $»^{29}$. Le bien que Machiavel a découvert, faut-il le répéter, porte sur la vérité effective du politique : «Dans les discours du livre précédent, ayant parlé des décisions que prirent les Romains sur ce qui se passait à l'intérieur de la cité, dans ce livre, nous parlerons de celles que prit le peuple romain sur l'augmentation de son empire ». En somme, en retournant vers la Rome ancienne, Machiavel enseigne, d'une façon tout à fait nouvelle, comment l' avenir pourrait être ${ }^{30}$. Du fait même, en faisant un travail d'historien, Machiavel marque l'histoire des idées.

Machiavel, avec d'infinies précautions, mais avec une volonté de fer, s'attaque à l'Église. La citation proposée ci-dessus montre l'auteur du Prince affrontant un prince de l'Église au sujet du pouvoir de l'Église justement, au sujet du pouvoir délétère de l'Église. Ce n'est qu'un exemple parmi d'autres de l'anticléricalisme plus-que-viscéral du Grand Secrétaire ${ }^{31}$. Car Machiavel est le premier penseur de la laïcisation radicale de la vie humaine et donc le premier moderne.

La pensée de Machiavel se déploie sur divers axes : celui de la verità effettuale ou de la vérité effective, celui de la fortuna ou des circonstances et celui de la tristezza ou de la méchanceté. Axe premier, axe des x, la vérité effective machiavélienne est doublement effective : elle est effective parce qu'elle est factuelle, parce qu'elle colle aux faits ; mais elle est aussi effective, en tant qu'efficace, c'est-à-dire parce qu'elle donne des résultats. Bien mieux, la vérité machiavélienne prétend être efficace parce qu'elle est factuelle, et inversement elle prétend rejoindre les faits parce qu'elle est efficace : elle permet de changer les faits, parce qu'elle s' appuie sur les faits. Par ailleurs, Machiavel présente la découverte de la vérité effective comme une conquête. Car il y a ce qu'on voudrait qui soit vrai et il y a ce qui est vraiment vrai, et l'un lutte contre l'autre dans le secret de tous les cœurs humains. Dans le Prince, Machiavel présente cet abîme épistémologique quatre fois de suite dans les chapitres seize à dix-neuf en coupant court chaque fois à l'illusion par une mise en garde. Un exemple :

Chacun comprend combien il est louable pour un prince de maintenir sa foi et de vivre dans l'intégrité et non astucieusement ; néanmoins, on voit par expérience que, de nos 
jours, ont fait de grandes choses les princes qui ont peu tenu compte de la foi et qui ont su avec astuce circonvenir les cervelles des hommes ; à la fin, ils ont prédominé sur ceux qui se sont fondés sur la loyauté. ${ }^{32}$

Or quand Machiavel se déplace du Prince pour aller vers les Discours, il reprend la même doctrine, avec des mots différents, par exemple, quand il s'agit de savoir comment les Romains créèrent l'institution des Tribuns. Machiavel établit alors un principe qui est accepté, dit-il, par « tous ceux qui raisonnent au sujet de la vie civile », un principe, dit-il, « dont toutes les histoires donnent plein d'exemples ${ }^{33}$. Il apparaît donc que raisonner sur la nature des choses et s'appuyer sur des exemples reviennne au même. Or ce principe doit guider « quiconque fonde une république et en organise les lois ». Ce que la raison et l'expérience enseignent sert directement à agir sur le monde, ici le monde politique. Machiavel place donc son lecteur sur le plan de la vérité effective ; il lui propose une connaissance factuelle et efficace.

Quel est ce principe effectif ? Il est assez terrible : tous les hommes sont méchants, et ils feront le mal chaque fois qu'ils en ont l'occasion et l'intérêt. Or l'homme de bonne foi et de bonne volonté protestera que cela est trop pessimiste ; s'il connaît la thèse épistémologique de Machiavel, il protestera aussi que l'expérience montre souvent que les hommes sont bons : après tout, peu de gens connaissent des tueurs, des voleurs et des violeurs ; et tous connaissent des hommes et des femmes qui sont révoltés par les criminels et leurs crimes. Or Machiavel emploie de nouveau, et de façon plus profonde, son concept de vérité effective : «Quand la méchanceté [des hommes] reste cachée un certain temps, cela vient d'une cause cachée qu'on ne connaît pas parce qu'on n'en a pas eu l'expérience. Mais le temps la découvre par la suite, le temps qu'on dit être le père de toute vérité » ${ }^{34}$. En somme, Machiavel proteste que la vérité effective est toujours là, même quand elle n'apparaît pas immédiatement au regard de tous. L'expérience est longue à acquérir parce que les faits se déploient dans le temps et parce que l'acquisition de l'expérience se fait dans le temps, mais surtout parce que les hommes se cachent la vérité par faiblesse, ou la cachent aux autres par ruse.

Mais alors comment distinguer entre la vérité effective cachée et l'illusion nourrie par l'imaginaire ? Il faut supposer que la vérité effective cachée est déjà visible pour celui qui sait regarder, et que la vérité effective difficile à voir ne fait que confirmer de visu ce qui a été vu ailleurs. Cette vérité effective cachée mais visible, incontournable en soi mais oubliable de fait, ne peut se trouver qu'en un lieu : l'expérience directe de ses propres désirs. Au fond, tous savent par expérience que les êtres humains sont tueurs, voleurs et violeurs parce que tous ont l'expérience de leurs désirs et de ce 
qui fait qu'ils cachent leurs désirs. Pour le dire autrement, même les préceptes de la religion rendent manifestes la vérité effective : «Tu ne tueras pas, tu ne voleras, tu ne désireras pas la femme d'un autre »sont des commandements qui nient ce qui est en autant qu'ils semblent supposer que l'homme peut être bon ; mais ces mêmes commandements signalent la vérité en rappelant ce qui est au fond de son cœur et qu'on réprime par des commandements, ici par les commandements de Dieu. Bien compris, ce qui devrait être se nie lui-même et pointe vers ce qui est. Soit la vérité effective ${ }^{35}$.

Encore une fois, quel est l'essentiel de cette vérité effective ? Que découvre-t-on quand on colle aux faits ? Deux choses. On apprend d'abord — c'est le deuxième axe, l'axe des y — que les circonstances ne sont rien de plus que des circonstances, qu'elles n'ont de profondeur ni anagogique ni eschatologique et donc qu' on peut, au moins en théorie, en faire ce qu'on veut. Si un homme est assez fort et assez intelligent, il saura modeler la fortune selon sa volonté, car il n'y a rien derrière la fortune. Cette double capacité humaine, morale et intellectuelle, Machiavel lui a donné un nom : la virtù, la vertu. En somme, la fortuna est par nature contrôlable par la virtù, et c'est l'essence de la virtù de pouvoir contrôler la fortuna. Et l'auteur du Prince a fixé pour toujours son idée sur la relation entre la fortune et la vertu grâce à une image saisissante. Il parle comme le ferait un homme de bon sens : «Il ne m'est pas inconnu que beaucoup ont eu et ont l'opinion que les choses du monde sont gouvernées par la fortune et par Dieu ${ }^{36}$. Il ne faut pas se méprendre sur le sens de la conjonction et. Dire «par la fortune et par Dieu », c'est dire que pour les gens de son temps, la vérité sur les circonstances est qu'elles sont en dernière analyse les instruments de Dieu. Mais Machiavel désacralise le monde en tant que projection de la volonté divine ${ }^{37}$ et, le mot n'est pas trop fort, viole ce qui en reste ${ }^{38}$.

Moi je juge qu'il est mieux d'être hardi que craintif, parce que la fortune est une femme et qu'il est nécessaire, lorsqu'on veut la garder sous contrôle, de la battre et de la bousculer. Et on voit qu'elle se laisse plutôt vaincre par ceux-ci que par ceux qui procèdent froidement ; c'est pourquoi, comme une femme, elle est toujours l'amie des jeunes, parce qu'ils sont moins craintifs, plus féroces, et qu'ils la commandent avec plus d'audace. $^{39}$

La même doctrine est présentée dans les Discours à partir d'une considération plus politiquement correcte. Examinant l'histoire de Rome dans son ensemble, Machiavel ressasse la question, devenue classique depuis Plutarque, de savoir si l'empire romain fut le résultat de circonstances, la fortuna, ou de la vertu, la virtù :

Nombreux sont ceux qui ont eu l'opinion, et parmi eux il y a Plutarque, un écrivain très sérieux, que dans l'acquisition de son empire le peuple romain fut plus favorisé par la 
fortune que par la vertu. [.. .] Il semble que Tite-Live se rallie à cette opinion, parce que, lorsqu'il fait parler un Romain de la vertu, il arrive rarement qu'il n'y ajoute pas la fortune. Moi je ne veux le reconnaître en aucune façon et je ne crois pas non plus que ça puisse se soutenir. [. . .] La vertu de ses armées lui fit acquérir son empire. [. . .] De telle façon que moi je crois que tous les princes qui procéderaient comme les Romains et auraient la même vertu qu'eux auraient la fortune qu'eurent les Romains. ${ }^{40}$

C'est répéter de façon académique et presque professorale le scandale qui éclata d'abord dans le Prince. Mais il y a en quelque sorte un autre scandale : Machiavel se dresse ici non seulement contre l'idée religieuse de la fortune, mais contre les autorités anciennes dont il a si souvent tiré profit ; en répudiant Dieu et l'Église, il répudie simultanément l'Antiquité et la philosophie ancienne. La disparition du Dieu chrétien est simultanément la disparition de l'expérience de ce que les Anciens appelaient tukhè, la déesse Fortune, force plus grande que les moyens humains.

Est-ce tout ce que contient la vérité effective ? Non certes : on y découvre l'autre scandale typiquement machiavélien, la tristezza humana, soit la méchanceté naturelle des hommes. C'est l'axe des z. Dans le Prince, parlant de la parole donnée, Machiavel conseille au prince de mentir pour garder le pouvoir : «Par conséquent, un seigneur prudent ne peut ni ne doit conserver sa foi, lorsqu'une telle observance se retourne contre lui et que sont anéanties les causes qui la lui firent promettre. Si les hommes étaient tous bons, ce précepte ne serait pas bon ; mais parce qu'ils sont méchants [tristi] et qu'ils ne te la conserveraient pas, toi non plus tu ne dois pas la leur conserver ${ }^{41}$. Machiavel ne prononce pas un jugement moral en parlant de la tristesse ou de la méchanceté des hommes. Pas plus qu'il ne s'effarouche à l'idée que le prince doive mentir, il ne dénonce que les hommes ne pensent qu'à eux. Pour le dire autrement, il reconnaît — car les faits le lui ont montré — que cette méchanceté est naturelle : s'en plaindre vaudrait autant que se plaindre de ce que le feu brûle. Au fond, parler de la méchanceté des hommes est une façon colorée de dire qu'ils sont égoïstes ${ }^{42}$. Encore une fois, cette vérité effective est difficile à accepter. Car qui veut avouer que l'amour est toujours amour de soi-même?

Et pourtant, écrit Machiavel, elle est la vérité au cœur des événements les plus importants de l'histoire, par exemple, le succès séculaire de Rome. Les humanistes italiens de la Renaissance avaient peint une image de l'harmonie romaine et du souci pour le bien commun qui animait soit les patriciens, soit les plébéiens, soit l'une et l'autre classes de la Rome républicaine. Dans les Discours, Machiavel s'inscrit en faux contre ceux qui ne reconnaissent pas la vérité effective au sujet de l'être humain : il y a toujours eu une tension fondamentale entre les patriciens et les plébéiens, tension qui explosait régulièrement en conflits politiques. Voilà pour les faits. Mais 
Machiavel ajoute comment on doit en juger en examinant si l'harmonie rêvée par les humanistes est possible : "Aussi il m'a semblé que c'est une chose digne de considération de voir si l'on pouvait organiser à Rome un régime qui se défasse des dites controverses ». Or, après une longue analyse où Machiavel compare Rome à Sparte, cet autre symbole de la perfection politique, il conclut :

\begin{abstract}
Mais toutes les choses humaines étant en mouvement et ne pouvant demeurer immobiles, il faut qu'elles montent ou qu'elles descendent. La nécessité vous contraint à de nombreuses choses auxquelles la raison ne vous pousse pas. [. . .] Il faut tolérer les inimitiés pouvant naître entre le peuple et le sénat, en les prenant comme un inconvénient nécessaire pour parvenir à la grandeur de Rome. ${ }^{43}$
\end{abstract}

Encore une fois, le réalisme de Machiavel le conduit à reconnaître que la violence entre les hommes est naturelle et qu'il serait illusoire de le nier. Bien mieux, les Discours prouvent au lecteur attentif que, selon l'auteur, la violence entre les classes de Rome fut le moteur qui fit la grandeur de Rome, laquelle grandeur se développa en faisant violence à toutes les sociétés qui entourèrent Rome ${ }^{44}$. Vouloir la grandeur humaine sans l'égoïsme ou la méchanceté humaine, c'est vouloir la beauté du feu sans oxygène pour le nourrir, sans bois à brûler.

Donc vérité effective, fortune à vaincre par la vertu et égoïsme humain. Ces trois axes, repensés à la lumière de l'anticléricalisme signalé plus haut, permettent pour ainsi dire de visualiser l'originalité de la position de Machiavel. Comme chacun le sait, du moins chacun qui se souvient de son petit catéchisme, le christianisme suppose lui aussi trois axes. Mais on ne les appelle pas des axes ; on les appelle des vertus théologales : la foi, l'espérance et la charité. Le chrétien croit en Dieu, certes. Mais il croit que Dieu est Providence, et donc il espère en Sa grâce. Et il croit que l'amour du prochain, pour l'amour de Dieu et par la grâce du Même est le moteur secret de l'histoire humaine. Donc fides, spes et caritas, d'un côté, verità effettuale, fortuna et tristezza, de l'autre. L'opposition est complète et symétrique ${ }^{45}$. En somme, guidé par Machiavel, l'homme, c'est-à-dire chacun de nous, est placé devant deux possibilités, ou deux interprétations du monde et de la vie : celle du christianisme et celle de l'homme du monde ${ }^{46}$.

Ces considérations philosophiques semblent bien loin de l'histoire, ou encore de l'histoire selon Machiavel ${ }^{47}$. L' Histoire de Florence de Machiavel permettra de retourner à ce qui semble avoir été quitté : les travaux de l'homme désœuvré que fut Nicolas Machiavel à la fin de sa vie. Qu'en est-il de l'historien qui fut un penseur politique ? Pour répondre à la question, il faudrait tenir compte des caractéristiques de cet étrange livre d'histoire qu'est l'Histoire de Florence. 
L'auteur lui-même révèle la première. Alors que Machiavel, à la demande de Julien de Médicis, écrivait une histoire de Florence qui était en large partie une présentation de la grandeur de la famille de Médicis ${ }^{48}$, il confiait à un proche le principe de lecture suivant.

Donato, je ne puis écrire l'histoire de Florence depuis le moment où Côme de Médicis s'empara de l'État jusqu'à la mort de Laurent de Médicis comme je l'écrirais si j'étais tout à fait libre. Les actions que je raconterai seront vraies et je n'en omettrai aucune ; j'éviterai seulement de disserter sur les causes universelles des événements. Ainsi je dirai ce qui arriva quand Côme s'empara de l'État, mais je tairai comment, par quels moyens, un particulier peut parvenir à s'élever de cette façon. Si quelqu'un veut m'entendre sur ce point, qu'il remarque bien ce que je ferai dire à ses adversaires : ce que je ne veux pas dire, moi, comme venant de moi, je le ferai dire à ses adversaires. ${ }^{49}$

Sans doute ce conseil de lecture est-il une indication du respect ironique dont Machiavel couvrait tous les hommes puissants, en l'occurrence le pape Clément VII, dont les aïeux étaient justement Côme et Laurent de Médicis ${ }^{50}$. Plus importante est l'indication que l'Histoire de Florence ne propose pas directement les thèses essentielles de la pensée de Machiavel : elle étale les faits, alors que les causes, c'est-à-dire les principes explicatifs du jeu historique, sont présentées discrètement. Il ne s'agit donc pas de chercher des analyses percutantes comme dans le Prince ou subtiles comme dans les Discours, mais des exemples qui sont des illustrations des thèses de Machiavel.

Cependant on ne peut pas s'en tenir à cette première remarque, car l'Histoire de Florence est construite selon un schéma net : les huit livres sont introduits par un premier chapitre qui offre des remarques générales sur la vie politique et le cœur humain ${ }^{51}$. Machiavel va jusqu'à reconnaître qu'il $\mathrm{y}$ a là une décision consciente, un choix d'écriture ${ }^{52}$. Ainsi le premier chapitre du livre deuxième porte sur les colonies, celui du troisième porte sur le conflit naturel entre les gens du peuple et les grands, et celui du cinquième sur le ramollissement moral et politique que cause la paix. Machiavel le penseur politique est donc tout à fait présent lorsque Machiavel l'historien écrit.

En revanche, il y a là un problème ; car le travail de l'un peut interférer avec le travail de l'autre. C'est le cas, par exemple, lorsque Machiavel dénonce à plusieurs reprises les guerres menées par des armées mercenaires : presque toujours il se plaint que les batailles n'ont fait que peu de morts parce que les institutions militaires en Italie étaient déficientes ${ }^{53}$. Ceux qui ont tenté d'excuser les inexactitudes de l'Histoire de Florence ${ }^{54}$ n'ont fait que souligner que l'historien florentin est plus près de son modèle, Tite-Live, que des règles de l'historiographie contemporaine ${ }^{55}$. On touche alors à une difficulté épistémologique de la pensée machiavélienne : Si la vérité fac- 
tuelle est le fondement de tout jugement solide, que faire des faits qui, au moins à première vue, ne collent pas au dur réalisme machiavélien ? Si on ramène certains faits sous la domination des autres, comment savoir lesquels sont les plus vrais? En somme, lorsqu' on a vu qu'un verre contient la moitié de l'eau qu'il peut contenir, comment savoir s'il est à demi plein ou à demi vide ? Les conclusions de Machiavel, et celles de ses adversaires, peuventelles prétendre être autre chose qu'un choix humain, trop humain?

Un passage de l'Histoire de Florence soulignera cette difficulté, puisque sa vérité historique est plus que problématique. Machiavel raconte une des actions politiques qui conduisirent à faire de Florence la ville des Médicis : la révolte du petit peuple contre les nobles. Pendant plusieurs nuits les citoyens les plus démunis se réunirent en cachette du pouvoir. Il arriva qu'un d'eux, « un des plus ardents et des plus expérimentés pour encourager les autres $»^{56}$, fit un long discours, qui est comme un tract machiavélien. Entre autres choses, il dit ceci : «Ceux qui vainquent n'en éprouvent jamais de la honte, quelle que soit leur façon de vaincre. Et nous ne devons pas tenir compte de la conscience, parce que là où, comme pour nous, il y a peur de la faim et de la prison, la crainte de l'enfer ne peut pas et ne doit pas gagner ${ }^{57}$. Mais si vous notez la façon de procéder des hommes, vous verrez que tous ceux qui parviennent à de grandes richesses et à un grand pouvoir y sont parvenus ou bien par la fraude ou bien par la force. Puis ces choses qu'ils ont usurpées par tromperie ou violence, ils les ont rendues honnêtes sous le faux nom de gain pour cacher la laideur de leur acquisition ». Quiconque connaît le Prince et les Discours reconnaît dans ces propos le machiavélisme le plus pur: les axes de la vérité effective, de vertu contre la fortune et de la méchanceté des hommes. Or Machiavel ajoute à la fin un commentaire qu'il revendique en tant qu'historien : «Ces arguments firent brûler fortement des esprits déjà chauds par eux-mêmes pour le mal ».

Pour être juste, il faudrait commenter ces paroles pendant longtemps. Une seule remarque servira d'entrée en matière, et de conclusion : Machiavel montre un orateur qui ne prouve pas, lentement, froidement, méticuleusement, la vérité de sa thèse ; il montre un homme qui ne fait que dire tout haut ce que les autres savent déjà par l'irrésistible désir du cœur humain, celui de survivre, celui de vaincre, celui d'arracher à l'autre ce qu'il faut pour soi. Sans doute, Machiavel se peint-il en peignant, plus exactement en inventant, cet orateur anonyme. Mais il croit aussi peindre les hommes en peignant la foule qui découvrit la vérité de ses désirs en entendant d'habiles paroles sans honte. Et enfin il dit quel est l'essentiel de son travail pédagogique : conduire les hommes à reconnaître ce qu'ils savent déjà, conduire les hommes à s'avouer ce qu'ils sont en leur disant tout haut ce qu'ils pensent tout bas. 
Ce que dit l'orateur de 1'Histoire de Florence, révèle non seulement la réponse machiavélienne, mais aussi la question à laquelle répond le Grand Secrétaire : qu'en est-il de l'être humain et de ses limites naturelles ou divines ? S'il est permis de se référer à Soljénitsyne, le conflit entre les prétentions de l'affirmation de soi moderne et l'autolimitation traditionaliste, fondée dans la prise de conscience que l'homme n'est qu'une partie du Tout, ce conflit est le problème par excellence de l'humanité, le problème qui est derrière les guerres mondiales et les camps de concentration du vingtième siècle et sans doute le conflit qui se trouvera au cœur de l'histoire du vingt et unième siècle. Énoncé si joliment par Machiavel qui invente un discours politique dans le traité historique qui est le sien, le problème théologico-politique n'est pas réglé au début du troisième millénaire, comme le prouveront les débats globalistes et régionalistes, laïcistes et intégristes, techno-scientifiques et environnementalistes à venir. Car la guerre civile mondiale n'a fait que commencer.

Collège de Sainte-Foy

\section{Notes}

1. Niccolò Machiavelli, Opere, éd. Sergio Bertelli et Franco Gaeta, 8 vol., vol. VI (Lettere, éd. Franco Gaeta), Milan, Feltrinelli, 1961, Lettre du 9 avril 1513. La traduction du texte de Machiavel est la mienne.

2. Lettre du 6 septembre 1520.

3. Discours, Lettre dédicatoire, Opere, vol. I (Il principe e Discorsi sopra la prima deca de Tito Livio, éd. Sergio Bertelli), Milan, Feltrinelli, 1960.

4. Montesquieu, De l'esprit des lois, XXIX, 19, Euvres complètes, Préface de Georges Vedel, Présentation et notes de Daniel Oster, Paris, Seuil, 1964, p. 755.

5. Lettre du 21 octobre 1502.

6. Voir Plutarque, Vie de Philopæemen, 3.1 à 4.10.

7. Prince, chapitre 14, Opere, vol. I.

8. Ibid., chapitre 15.

9. Pour la vie de Machiavel, consulter l'incontournable Vita di Niccolò Machiavelli de Roberto Ridolfi, 7 éd., rév., Florence, Sansoni, 1978.

10. Les célèbres Ghiribizzi, adressés à la famille de Piero Soderini, permettent de mesurer la taille relative du gonfalonier et de son secrétaire. Voir Ridolfi, Vita di Machiavelli, p. $149-56$.

11. Histoire de Florence, Lettre dédicatoire, Opere, vol. VII (Istorie fiorentine), Milan, Feltrinelli, 1961. Voir plus bas sur la droiture du récit historique de Machiavel.

12. Discours I, Proème.

13. Comme on le signalera plus bas, les deux premières causes ne doivent pas être éliminées de l'analyse machiavélienne. 


\section{Voir Discours I, 39.}

15. Avec des résultats insatisfaisants, comme le prouve la vie de Machiavel. Pour la petite histoire, on notera l'anecdote que rapporte son biographe : « Au même moment, on lui [Laurent de Médicis] donna une paire de chiens de chasse ; il montra plus beau visage et dit plus belles paroles à celui qui lui avait donné les chiens qu'à lui [Machiavel]. Aussi il [Machiavel] partit de là insatisfait. » (Ridolfi, Vita di Machiavelli, p. 258).

16. Prince, Lettre dédicatoire.

17. Ibid., chapitre 14 .

18. Sur le thème de l'éducation par l'histoire, voir Discours II, 2 fin.

19. Le chapitre vingt-six livre sans doute l'intention politico-politique la plus profonde qui préside à l'œuvre : le Prince peut inspirer les princes à accomplir les plus grands projets politiques, ceux des fondateurs de peuple.

20. Quelques phrases, très personnelles, de Machiavel indiquent qu'il se voit, au cœur même de son exil forcé, comme un homme dont la seule nourriture est la pensée. Mais la pensée n'exclut jamais chez lui le devoir de l'action, fût-ce bêtement de rouler une pierre, comme il dit. Voir la lettre du 10 décembre 1513 à Francesco Vettori.

21. Le consensus des commentateurs là-dessus est sujet à caution. Voir Discours II, 1, mais en le comparant à III, 42.

22. Prince, chapitre 15.

23. Ce qui ne signifie pas que Machiavel ne visait pas, en même temps, d'autres institutions, bien historiques celles-là, qu'il avait vues « exister dans la réalité » mais pour dépérir promptement, par exemple, la république de Florence, consacrée au Christ, dont Savonarole fut le maître. Elle tomba juste avant que Machiavel ne soit engagé comme secrétaire de la Seconde Chancellerie. Or la république savonarolienne était une tentative de fondre, et de fonder sur le sol de Florence, la république de Platon et le Royaume des cieux. Voir Roberto Ridolfi, Vita di Girolamo Savonarola, 2 vol., Rome, Belardetti, 1952, chapitres 10 et 13 (t. 1, p. 127-44 et 180-95). Pour l'avis de Machiavel sur Savonarole, voir Prince, chapitres 6 et 12 .

24. Voir Prince, chapitres 14, 17 et 18 pour une critique de Xénophon, de Tite-Live et de Cicéron. Voir aussi le chapitre 25, où Machiavel s'oppose à l'opinion commune de son temps.

25. Tâche suprêmement difficile, comme il le reconnaît : «On doit considérer qu'il n'y a rien de plus difficile à traiter, ni plus douteux quant à la réussite, ni plus dangereux à manœuvrer, que de se faire chef pour introduire de nouvelles institutions parce que l'introducteur a pour ennemis tous ceux qui tirent profit des vieilles institutions et a pour défenseurs tièdes tous ceux qui bénéficieraient des nouvelles institutions. »(Prince, chapitre 6). Voir aussi Discours III, 35.

26. Et elle l'est parce que le mot anima, âme, est banni du Prince et des Discours pour ne laisser qu'animo, mot beaucoup plus terre à terre, qui équivaudrait à notre cerveau. Pour l'emploi du mot cerveau, voir Prince, chapitre 18.

27. Discours I, Proème.

28. Qu'il ne soit pas le seul de son temps à le penser et que son statut d'éducateur ait été très précoce, on le comprendra par ce qui suit : «L'influence de Machiavel pénètre en Angleterre avec Thomas Cromwell [donc peut-être dès 1515, soit immédiatement après l'écriture du Prince et dix-sept ans avant la publication du livre], dont la politique fut soigneusement calquée sur les principes du penseur florentin. Thomas Cromwell conseilla à Reginald Pole 
d'utiliser comme manuel politique le Prince, qu'il possédait en manuscrit bien avant la publication qui en fut faite en Italie, et qu'il considérait comme un ouvrage sur le gouvernement bien plus utile que les rêves de Platon. » (Lewis Einstein, The Italian Renaissance in England, London, Macmillan, 1902, p. 291-92 ; la traduction est la mienne). La lecture du premier livre de l'Utopie de More, publié en 1516, servira de signe supplémentaire, mais a contrario, de l'entrée de l'œuvre de Machiavel en Angleterre.

29. Discours II, proème.

30. Ces remarques sont bien sérieuses, et Machiavel ne l'était pas tout le temps. Il aimait même mêler les remarques scabreuses aux réflexions respectables. Comme il l'écrivait à son ami Francesco Vettori, «Qui verrait nos lettres, honorable compère, et en verrait la diversité, serait fort étonné, car il lui semblerait tantôt que nous sommes des hommes graves, tout à fait tournés vers de grandes choses, et que de nos seins ne peut tomber aucune pensée qui n'ait en elle de l'honnêteté et de la grandeur. Mais, tournant ensuite la page, il lui semblerait que nous, les mêmes, sommes légers, inconstants, lascifs et tournés vers des choses vaines. » (Lettre, 31 janvier 1515). Puis ayant écrit cela, Machiavel quitta ses considérations sur les aléas de sa vie amoureuse pour aborder les intrigues de la politique italienne. Or on pourrait faire le chemin inverse avec lui et aller de la philosophie politique à la philosophie de l'amour.

31. La toute première lettre de Machiavel, du moins la toute première que l'histoire a conservée, porte sur Savonarole : le futur auteur du Prince se moque des manigances politico-religieuses du père dominicain. Voir la lettre du 9 mars 1498 à Ricciardo Becchi. De plus, dans les Discours (I, 27), Machiavel conclut au sujet de l'occasion que rata Baglioni d'assassiner le pape Jules II : «Les hommes ne savent être ni honorablement mauvais ni parfaitement bons. Si une mauvaise action a de la grandeur ou est d'une certaine manière généreuse, ils ne savent pas l'accomplir ». Voir aussi Histoire de Florence I, 9. Enfin, dans l'Histoire de Florence (III, 7), il loue le peuple de Florence lorsqu'il résista aux excommunications générales du pape Grégoire VII par ces mots qui en disent long sur sa pensée : «Ces citoyens respectaient plus leur patrie que leur âme ». Voir aussi la lettre du 16 avril à Francesco Vettori.

32. Prince, chapitre 18.

33. Discours I, 3.

34. Ibid.

35. Un auteur aussi chrétien que Pascal peut être un parfait machiavéien quand il s'agit de sonder le cœur humain et d'en tirer les conclusions effectives : «Tous les hommes se haïssent naturellement l'un l'autre. On s'est servi comme on a pu de la concupiscence pour la faire servir au bien public. Mais ce n'est que feindre et une fausse image de la charité, car au fond ce n'est que haine. » (Blaise Pascal, Pensées, éd. Michel Le Guern, 2 vol., coll. «Folio », Paris, Gallimard, 1977, 196, Br. 451, t. I, p. 166).

36. Prince, chapitre 25.

37. Après avoir traité de Dieu, selon ce que les autres croient, il écrit, en son propre nom, ce qui suit : « Néanmoins [...] je juge qu'il peut-être vrai que la fortune soit l'arbitre de la moitié de nos actions, mais que même alors elle nous laisse gouverner l'autre moitié, ou à peu près. » Lorsque Machiavel lui-même se prononce, Dieu disparaît. Ou plutôt il l'évince. Bientôt, inoccupé, Il disparaîtra tout à fait, comme nous l'apprend Nietzsche : Dieu est mort parce que les hommes L'ont éliminé de leurs vies. 
38. Avec des précautions louables, ou du moins admirables, Descartes opère une désacralisation semblable de l'ensemble de la nature dans son Discours. Voir, entre autres, les paragraphes deux et trois dans la cinquième partie du Discours de la méthode.

39. Prince, chapitre 25. Le Prince offre donc au lecteur une autre version de l'histoire de la séduction de Lucrezia par Callimaco ; ou encore la Mandragore est la version comique du Prince. Pour comprendre comment un dramaturge pourrait répondre à Machiavel, il faut lire l'Othello de Shakespeare en considérant la possibilité que la relation entre Iago et Roderigo est la vérité de la relation entre Ligurio et Callimacco. Mais alors la mort de l'épouse et le suicide de l'époux sont les effets réels de l'entreprise menée par Iago-Ligurio.

40. Discours II, 1.

41. Prince, chapitre 18.

42. «Ne nous emportons point contre les hommes en voyant leur dureté, leur ingratitude, leur injustice, leur fierté, l'amour d'eux-mêmes, et l'oubli des autres : ils sont ainsi faits, c'est leur nature, c'est ne pouvoir supporter que la pierre tombe ou que le feu s'élève. » (Jean de La Bruyère, Caractères ou les Moeurs de ce siècle, éd. Robert Garapon, Paris, Garnier, 1962, De l'homme, p. 296). Voilà, sans doute, pourquoi le mot charité apparaît une seule fois dans le Prince, et ce dans un contexte qui lui donne le sens d'amour de soi. Pour la description de la relation sans pitié ni admiration entre le prince et ses ministres, voir Prince, chapitres 22 et 23 .

43. Discours I, 6 .

44. C'est déjà la thèse avancée en Discours I, 4. Voir aussi I, 16 et 46, et II, 8. Voir aussi Histoire de Florence II, 12, III, 1 et IV, 1.

45. Pour saisir synthétiquement cette opposition, voir l'emploi du mot péché au chapitre 12 du Prince. Voir aussi Discours II, 18.

46. Ce choix, Machiavel l'a illustré dans sa Vie de Castruccio Castracani. Selon ce récit, le jeune Castruccio, fils adoptif d'un prêtre, eut un jour à répondre à François Guinigi, condottiere célèbre de sa ville : « Il l'appela un jour et lui demanda où il resterait plus volontiers : dans la maison d'un gentilhomme, qui lui apprendrait à monter à cheval et à pratiquer les armes, ou dans la maison d'un prêtre, où il n'entendrait rien d'autre que des offices et des messes. » Héros selon le cœur de Machiavel, Castruccio répondit, on le devine, à l'appel de sa nature véritable et non à celle de l'éducation qu'il reçut de son père adoptif. Mais en changeant de mode de vie et donc de mode de pensée, il changea pour ainsi de mode d'être : il « passa donc de la maison de messire le chanoine Antoine Castracani à la maison de messire le condottiere François Guinigi : il est extraordinaire de penser en combien peu de temps il se remplit de toutes les vertus et manières qu'on requiert d'un véritable gentilhomme. » (Vie de Castruccio Castracani, vers le début ; la traduction est la mienne). La morale de la fable machiavélienne est la suivante : pour devenir un véritable gentilhomme et donc un homme selon la nature, il faut d'abord reconnaître et prendre le chemin de la vérité et donc quitter les illusions qui passent pour la voie de la vérité.

47. Pour compléter le tableau il faut sans doute y ajouter et faire de ce diptyque un triptyque. Car le réalisme machiavélien anti-chrétien est en même temps un refus d'un mode de vivre et de penser qui précéda le christianisme : ce qu'on appelle la pensée de l'Antiquité. Les axes de la pensée ancienne ne sont pas la foi, l'espérance et la charité, mais l'idea ou la loi éternelle derrière le monde réel, l'éudaimonia ou le bonheur de l'âme comme fin naturelle de l'homme et l'éros ou l'élan naturel qui est au cœur des choses et au cœur du cœur humain. Voir, par exemple, Platon, Banquet, passim. 
84 / Renaissance and Reformation / Renaissance et Réforme

48. Le livre atteint une double apothéose lors de deux longues tirades qui résument la vie des plus grands de Médicis : Côme et Laurent le Magnifique. Voir VII, 6 et VIII, 26.

49. Ridolfi, Vita di Machiavelli, p. 310. Voir aussi la lettre du 17 mai 1521 et la lettre du 30 août 1524 à Francesco Guicciardini.

50. Loin de voir une lâcheté dans cette prudence pédagogique, Machiavel y voyait un principe même du travail d'écriture des historiens. La reconnaissance de cette prudence devient du fait même un principe de lecture des historiens. Voir Discours II, 10 et III, 2.

51. Il n'y a qu'une exception, le premier livre, dont le chapitre théorique est sans doute l'avant-propos.

52. Par exemple, il souligne les thématisations contenues dans le premier chapitre des livres VII et VIII au moyen des expressions suivantes : « selon notre habitude » et « selon notre coutume».

53. Voir par exemple, VII, 20. Par ailleurs, le chapitre VI, 34 décrit méticuleusement une tornade, qui aurait été l'œuvre de Dieu. C'est le seul exemple d'événement naturel important dans l'Histoire de Florence.

54. Voir par exemple, Ridolfi, Vita di Niccolò Machiavelli, p. 307 : « Il écrit l'histoire plutôt en tant que penseur politique qu'en tant qu'historien. C'est pourquoi ne lui importe pas la recherche diligente et minutieuse des faits qui rendra célèbres les histoires plus vraies de Guicciardini. Il veut tirer des faits des enseignements, des normes, des doctrines, et il ne se fera pas un scrupule d'adapter quelques fois les faits aux doctrines. »

55. Sur la reconstruction historique que Tite-Live lui aussi aurait osé faire, voir Discours II, 6. Voir aussi Histoire de Florence VI, 20.

56. Histoire de Florence III, 13.

57. «ne peut pas et ne doit pas ». Voilà rendu visible, en une seule tournure, tout le mystère de la pensée réaliste, et peut-être de la pensée moderne : si une chose ne peut pas gagner, parce qu'elle n'est pas réelle, pourquoi ajouter qu'elle ne doit pas gagner ? quelle crainte peut-on avoir que le réel — ce qui ne peut pas ne pas être — ne soit pas, et surtout qu'il ne soit pas à cause de ce qui n'est pas? 